\title{
Analysis of Isothermal Physical Aging of Polycarbonate from Tensile Creep Tests
}

\author{
Sudheer Kolisetty ${ }^{1}$, Rajeswara Reddy Resapu ${ }^{2}$ \\ '(Department of Mechanical Engineering, Gitam University, India) \\ ${ }_{2}^{2}$ (Department of Mechanical Engineering, Gitam University, India)
}

\begin{abstract}
Physical aging behavior of polycarbonate has been determined by performing a series of tensile creep tests at different temperatures. A series of short-term creep tests along with finite amount of relaxation time in between the creep tests were performed in a sequence on a thin film of polycarbonate at a temperature below its glass transition temperature $\left(T_{g}\right)$. The sequence of tests was repeated at 3 different temperatures. Compliance during each creep test was measured and this data was fitted using Kohlraush functions. All the Kohlraush curves are then shifted on to a reference curve on a log-time scale using a program called Phyage. The amount of shift or shift factors of each Kohlraush curve onto the total reference curve are used to determine the shift rate at that particular temperature. The shift rate is used to predict of the amount of physical aging experienced by the material at that particular temperature. The shift rate of the polycarbonate film is determined at three different temperatures.
\end{abstract}

Keywords: Physical Aging, Creep, Viscoelasticity, Compliance, Polycarbonate

\section{Introduction}

Physical aging refers to structural relaxation of the unstable glassy state towards a stable equilibrium state, and it is accompanied by changes in almost all physical properties. This term was first proposed by Struik [1] to distinguish relaxation from the glassy state with other time-dependent processes such as recrystallization and chemical degradation. Physical aging is a time dependent phenomenon that is due to the gradual evolution of a glassy material below the glass transition temperature $\left(\mathrm{T}_{\mathrm{g}}\right)$ towards thermodynamic equilibrium. During this period, a number of properties vary continuously. Physical aging is particularly important for polymers, because the rate of physical aging is determined in part by how far below the glass transition temperature $\mathrm{T}_{\mathrm{g}}$ the polymer is used, and $T_{g}$ values are usually low for polymers. During Physical aging, changes in physical properties due to structural relaxation of the material occur. Physical aging also affects the characteristic time scale of dynamic properties which are important for a polymer. Coming to Polycarbonate materials, they are high performance, non-metallic materials. However, the properties of polymeric materials are somewhat different from metals because of the inherently different molecular structure which is strongly viscoelastic and its properties are time and temperature dependent. The behavior of viscoelastic polymers is characterized by their reaction to external loads and their reaction to external loads can be characterized in two ways. Under constant strain, the timedependent stress response is known as stress relaxation, while time-dependent strain response under constant stress is referred to as creep. Metals (and alloys) exhibit creep deformation at high temperatures but the difference in polymers is their ability to partially recover creep strain upon the removal of the load. The creep and stress relaxation behavior of a viscoelastic polymer can be illustrated by various responses of material to the applied load as shown in Figure 1. One of the physical properties that are used to characterize the material behavior is tensile compliance. Compliance is characterized as the ratio of strain to stress which is the inverse of modulus.

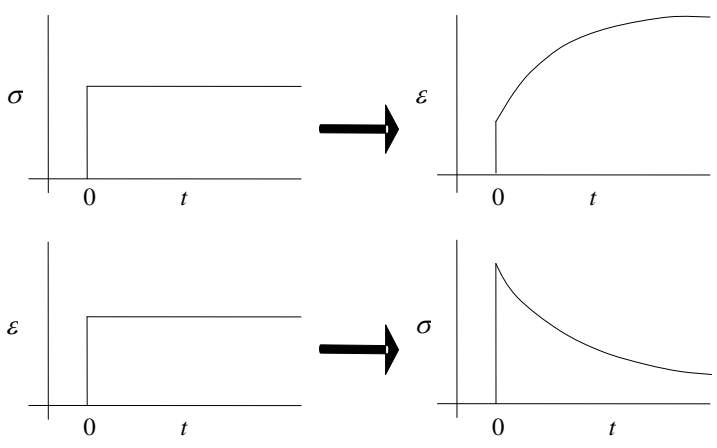

Figure 1. Creep and Stress relaxation behavior of a viscoelastic material 
Primary work on physical aging was done by Struik [1], who presented a framework that describes the interrelations of the various orientation effects (anisotropy, birefringence, etc.) and their dependence on the thermomechanical history of the material. The residual thermal stresses generated by rapid inhomogeneous cooling through the glass transition range were also discussed. The theory of the quenching of flat glass plates was described, generalized to objects of arbitrary geometry, and shown to be applicable to polymers. In a later work, the volume relaxation and simultaneous changes in mechanical properties that occur after complicated thermal histories are established by Struik [2]. It was shown that, for polystyrene, poly (vinyl chloride) and polycarbonate, the mechanical creep properties change as if the position on the log time scale of the creep curve is uniquely determined by the momentary value of specific volume. Thus, a maximum in the volume relaxation curve implies that the creep curve first shifts to the left and later to the right. Ferry [3] explained in detail, the viscoelastic behaviour of polymers reflecting the combined viscous and elastic responses, under mechanical stress, of materials which are intermediate between liquids and solids in character. Sullivan et al. [4] presented the linear viscoelastic creep behaviors of a unidirectional fiber reinforced plastic and its corresponding resin with special emphasis on elucidating the influence of physical aging. It was found that physical aging causes a significant reduction in the creep of these systems and that its influence is comparable in magnitude to that of the temperature. The temperature and aging dependencies of the viscoelastic shift rate, an important quantity needed for long term viscoelastic predictions, were discussed. Further, the time-temperature superposition principle is shown to be unsuitable when applied to long term creep results but is perfectly applicable to momentary creep results. Lawrence et al. [5] dealt with the expanded treatment of the behaviour of polymers with regard to material variables and test and use conditions. The general principles, useful empirical rules and practical equations were highlighted. Detailing the specific behaviour of many common polymers, they placed the emphasis on time and frequency dependence over temperature dependence, used contemporary molecular mechanisms to explain creep, stress relaxation, constant strain rate responses and crazing, provided explicit equations to predict responses, supplied a discussion of large deformation multiaxial responses, compared statistical and continuum theories on the same data set, and updated the stress-strain behaviour and particulate filled systems. Wang et al. [6] studied the Physical aging behavior of high-performance composites using tensile creep tests. They used effective time theory (ETT) to modify time/temperature superposition principle (TTSP) and incorporate physical aging effects. Brinson and Gates [7] described the effect of physical aging on long term creep of polymers matrix composites. They laid out, in a self-consistent manner, the basic concepts describing physical aging of polymers. Several aspects of physical aging which have not been previously documented are also explored in this study, namely the effects of aging into effective equilibrium and a relationship to the timetemperature shift factor. The physical aging theory is then extended to develop the long term compliance/modulus of a single lamina with varying fiber orientation. The latter is then built into classical lamination theory to predict long time response of general laminated composites. They illustrated that the long term response can be counter-intuitive, stressing the need for consistent modeling efforts to make long term predictions of laminates to be used in structural situations. Bradshaw and Brinson [8] developed a method for analysis using time-aging time superposition. They developed an analytic method based on error minimization and introduced the concept of a total reference curve as an alternative to choosing a single momentary curve for the reference. They concluded that most of the materials studied, the total reference using horizontal shifting alone is the recommended method; in the few remaining cases, the total reference procedure with both horizontal and vertical shifting resulted in the best outcome. O'Connell et al. [9] studied large deformation response of polycarbonate using time-temperature, time-aging time, and time-strain superposition and determined the type of superposition best suitable to the available data. They found that temperature and aging time could be described using a stretched exponential form relaxation function, and time-aging time superposition was found to be applicable to the data under all test conditions. However, the double logarithmic aging time shift rate, $\mu$ was found to vary significantly with both temperature and strain. They also found that, in both the time-aging time and time-temperature superposition analyses it was found that the application of vertical shifts was required for superposition of data. Masumoto [10] studied the time-temperature superposition and Physical aging in amorphous polymers. He noted that as ductile amorphous thermoplastics physically age below $\mathrm{T}_{\mathrm{g}}$, the structure of the polymer changes on a time scale comparable to the time duration of the creep test. He concluded that time-temperature superposition prediction greatly exaggerates the amount of creep in amorphous thermoplastics. He also concluded that for samples aged at the test temperature for one hour before testing, the difference between the time-temperature superposition prediction and the actual creep data after 10 days is greater than a factor of ten in time. Hutchinson et al. [11] studied the Physical aging of Polycarbonate by analyzing the effect of annealing polycarbonate at single temperature less than $\mathrm{T}_{\mathrm{g}}$ for different aging times. They compared the kinetics of the enthalpy relaxation process with the effects of aging at the same temperature on the creep response and on the yield behavior. Biswas et al. [12] studied creep behavior of glass fiber reinforced polycarbonate at elevated temperatures. They introduced two shift factors - a modulus shift factor and a time shift factor to draw the grand master curve of the creep compliance curve of various fiber contents. They 
concluded that it is possible to estimate the creep behavior of GFRPC composites with any fiber contents using the grand master curve of creep compliance master curves and time and modulus shift factors. Cangialosi et al. [13] studied the Physical aging of Polycarbonate by representing the free volume relaxation of Polycarbonate (PC) as a function of aging time at three different temperatures below $\mathrm{T}_{\mathrm{g}}$. A decrease in the number of cavities in the system was observed. The process was successfully modeled according to the Struik model, which describes a relaxation time dependent on both the temperature and the distance from equilibrium. Soloukhin et al. [14] used depth sensing indentation (DSI) to study the long term physical aging of Polycarbonate. The endothermic peak of polycarbonate shifted towards higher temperatures upon aging at an elevated temperature and did not change its location upon aging at room temperature. The elastic modulus and hardness of polycarbonate increased in a stepwise fashion during aging at room temperature. The changes in the mechanical properties appeared to have a reasonable correlation with free volume relaxation of the polymer, whereas the changes in the endothermic peak may be associated with internal energy changes. Barbero et al. [15] developed a low-cost, short-term testing procedure to predict the time dependent properties of polymer liners over the lifetime of the installation. Sakai et al [16] studied the creep behavior of a viscoelastic shape memory alloy using Physical aging. Short term creep tests were used to obtain the master curve for the creep compliance, and the time-temperature superposition principle was confirmed. The effect of physical aging depended on the time and temperature, confirming the time-physical aging time superposition principle, and achieved the effect of physical aging described as the aging shift rate. Using the master curve, time-temperature shift factor, and aging shift rate, creep deformation, including the effect of physical aging on creep behavior was estimated. In a later work, Sakai et al. [17], analyzed creep behavior in thermoplastics using Visco-elastic theory. They proved that, it should be possible to control the creep deformation by controlling temperature, the amount of fiber volume fraction, the effect of physical aging and the amount of crystallinity of a polymer. They also studied the effect of thermal histories on the creep compliance of Polycarbonate [18]. For the work in this paper, the procedure adopted by Resapu [19] has been followed, in which creep compliance at different aging times were used for predicting shift rate. However, instead of using the best set of data as the reference curve, the creep test with the most aging data is used as a reference curve. The details of the procedure followed are explained in detail.

Compliance behavior of a viscoelastic material can be characterized by Kohlraush functions [1]. The Kohlrausch function defining compliance for a viscoelastic material is given as:

$$
D(t)=D_{0} e^{(t / \tau)^{\beta}}
$$

where $t$ is the time during the test, $D_{0}$ is the initial (elastic) tensile compliance, $\tau$ is the relaxation time and $\beta$ is the power term. For a given initial condition $D_{0}$, the Kohlrausch function is capable of taking on a wide variety of shapes (by varying the parameter $\beta$ ) with a single relaxation time parameter $(\tau)$. Given the small number of parameters, the Kohlrausch function is very compact yet it is capable of fitting a wide variety of compliance behavior. However, stress/strain solutions using this function virtually never simplify due to the power term $\beta$.

Creep tests are performed following a standard test procedure has been developed by Struik [2]. In this method a series of short term creep tests are performed at certain intervals. Short term tests are defined as those whose duration is very short when compared to the aging time at the beginning of the test; as such, ongoing aging during each test can be neglected with aging considered approximately constant. Thus, the results obtained in each test represent the results corresponding to a particular aging time. A number of short term tests are performed on the same specimen at regular intervals of aging time and the momentary response function from each short term test is determined. The methodology of performing the short term tests has been established by Struik and it is shown in Fig. 2. The figure shows a creep load being applied on the specimen after regular intervals of time $\mathrm{t}_{\mathrm{e} 0}, 2 \mathrm{t}_{\mathrm{e} 0}, 4 \mathrm{t}_{\mathrm{e} 0}$ and $8 \mathrm{t}_{\mathrm{e} 0}$ and maintained for $0.1 \mathrm{t}_{\mathrm{e} 0}, 0.2 \mathrm{t}_{\mathrm{e} 0}, 0.4 \mathrm{t}_{\mathrm{e} 0}$ and $0.8 \mathrm{t}_{\mathrm{e} 0}$ respectively. The strain response of the specimen is considered to obtain compliance. It can be seen that the strain due to the current load is the total strain minus the extrapolated strain that would have occurred had the current load not being applied. The period of load is generally $1 / 10^{\text {th }}$ the aging time when the load is first applied. The strain and load data is then used to calculate the compliance for each short term test. The momentary response curves (compliance curves) obtained at different aging times are plotted on a log time scale; the response during the unloaded relaxation period of the aging phenomenon is not considered in this standard approach. The short term compliance obtained from each test can be superposed by horizontal shifting if the material is a thermorheologically simple material. In this case, the short term compliance $(D)$ is related to a single reference compliance curve $\left(D_{\text {ref }}\right)$ by the equation:

$$
D\left(t ; t_{e}, T\right)=D_{\text {ref }}\left(a_{t e} t ; t_{\text {eref }}, T\right)
$$

where $t_{\text {eref }}$ is the isothermal aging time at which the reference curve was defined, $t_{e}$ is the aging time at which the short-term test is taking place, $T$ is the isothermal temperature (below $\mathrm{T}_{\mathrm{g}}$ ) at which the short term tests took place and at which the reference curve is defined and $a_{t e}$ is the horizontal shift factor due to aging. As mentioned previously, vertical shifting can also be employed but is not used during this thesis. The shift factors required to superpose all the momentary curves horizontally onto one curve are determined. The shift factors determined 
are related to a known function of aging time in order to obtain a continuous prediction of shift factors at all aging time. The horizontal shift factor function is given by:

$$
a_{t e}=\left(\frac{t_{e r e f}}{t_{e}}\right)^{\mu}
$$

where $\mu$ is the horizontal shift rate. The shift factors decrease monotonically with the aging time (for isothermal tests) until the material reaches equilibrium, after which the shift factors theoretically remain constant. Given the test temperature and time scale, it is assumed that all of the tests are performed before the material has reached equilibrium. The shift factors thus form a line when plotted as log shift factor versus log aging time and the slope of that line in is given by $-\mu$. Once the compliance data is collected from a series of creep tests, the program PHYAGE [8] is used for data reduction of a typical set of time-aging time data. This includes fitting each of the experimental compliance data file with an appropriate compliance function, finding the optimal reference curve and the shift rates, and the associated shift factor function. The final output of the program is the total reference curve, a parameter called the shift rate which describes the shift factor function, and a prediction of the long term compliance of the material. The input to PHYAGE is a set of data files each containing the experimental compliance data from its associated short term creep test. PHYAGE first fits each set of data with an optimal Kohlrausch curve. These curves are called the momentary curves (MCs). It has been demonstrated by Struik [2] that for a thermorheologically simple material, these MCs can be superposed onto a single momentary master (reference) curve (MMC) at a reference aging time $t_{\text {eref }}$. This superposition can be performed by shifting all the MCs horizontally (in log time) onto the MMC.

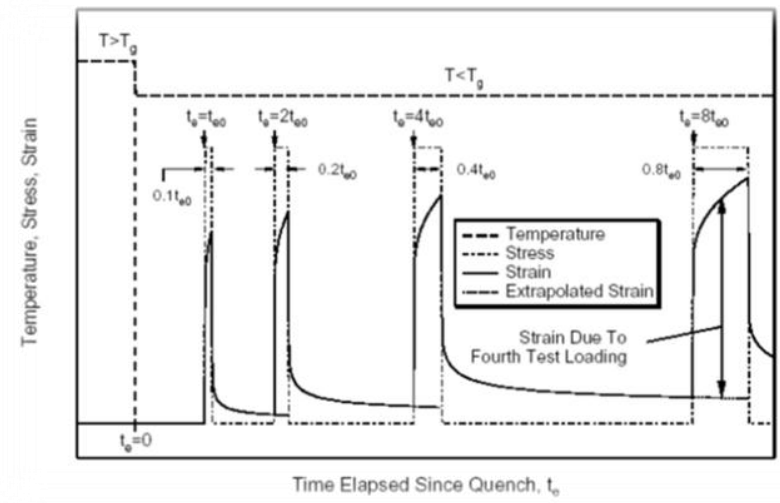

Figure 2. Isothermal aging test as developed by Struik (from [8])

\section{Experimental Details \& Results}

The material used during this experiment is the Polycarbonate film LEXAN 8010 of 0.007 inch thickness from the GE Structured Products. This material is high heat resistance, superior dimensional stability, and excellent clarity in all thicknesses and provides high gloss surface finishes while meeting additional requirements for added UV stability. For tensile testing, specimens of $1 \mathrm{in} \times 0.5 \mathrm{inch}$ height are prepared. To perform, isothermal aging tests, $\mathrm{T}_{\mathrm{g}}$ of the material need to be determined. DMA was used to perform a dynamic temperature sweep test to measure the storage modulus (E'), loss modulus (E') and the loss factor (tan $\delta$ ) as shown in Fig. 3. The temperature where the E' begins to drop or the temperature where 'tan $\delta$ ' hits its peak can be considered as the $\mathrm{T}_{\mathrm{g}}$ of the material. In this case, the $\mathrm{T}_{\mathrm{g}}$ of the material can be concluded to be in the range of $140-148^{\circ} \mathrm{C}$. Hence, it was decided to perform the Physical aging at temperatures below the determined range. Three temperatures, $120^{\circ} \mathrm{C}, 125^{\circ} \mathrm{C}$ and $130^{\circ} \mathrm{C}$ were chosen to perform creep tests. The test procedure established by Struik [1] as shown in Fig. 2 was followed. Hence, after heating the specimen to a temperature above $\mathrm{T}_{\mathrm{g}}$ (to about $160^{\circ} \mathrm{C}$ ), the temperature was suddenly brought down to the designated temperature $\left(120^{\circ} \mathrm{C}\right.$, $125^{\circ} \mathrm{C}$ or $130^{\circ} \mathrm{C}$ ). The aging time was calculated from that point. Once, the aging was commenced, short term creep tests were performed at intervals of $15 \mathrm{~min}, 30 \mathrm{~min}, 1 \mathrm{hr}, 2 \mathrm{hrs}, 4 \mathrm{hrs}, 8 \mathrm{hrs}$ and $16 \mathrm{hrs}$, with durations of $90 \mathrm{sec}, 180 \mathrm{sec}, 360 \mathrm{sec}, 720 \mathrm{sec}, 1440 \mathrm{sec}, 2880 \mathrm{sec}$ and $5760 \mathrm{sec}$ respectively. These times are considered to be short enough to neglect the ongoing aging during the load times (constant aging time for each test). The force for the creep test was selected as 1200 grams. This was determined after conducting a series of linearity tests at various load levels. Once all the creep tests were performed, the stress strain data during loading was used to calculate the compliance during each test. The experimental compliance data at $120^{\circ} \mathrm{C}$ was calculated and plotted on a log-time scale as shown in Fig. 4. 


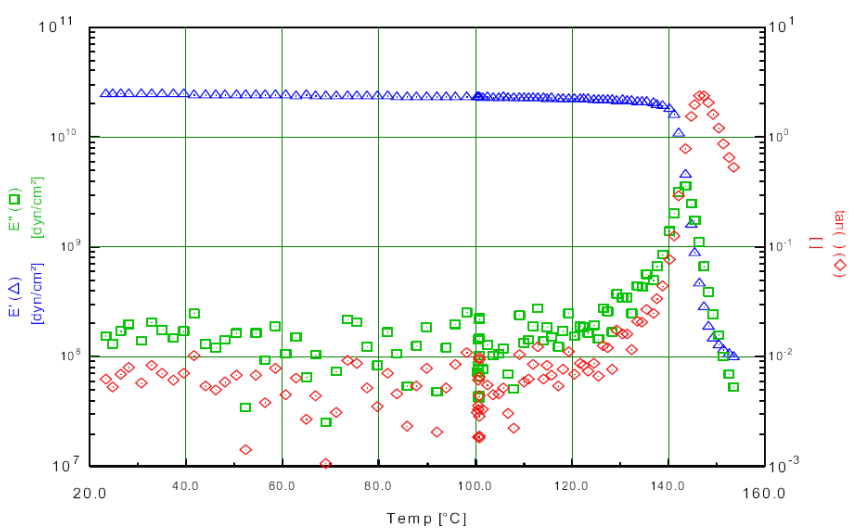

Figure 3. Dynamic Temperature ramp test to determine $T_{g}$

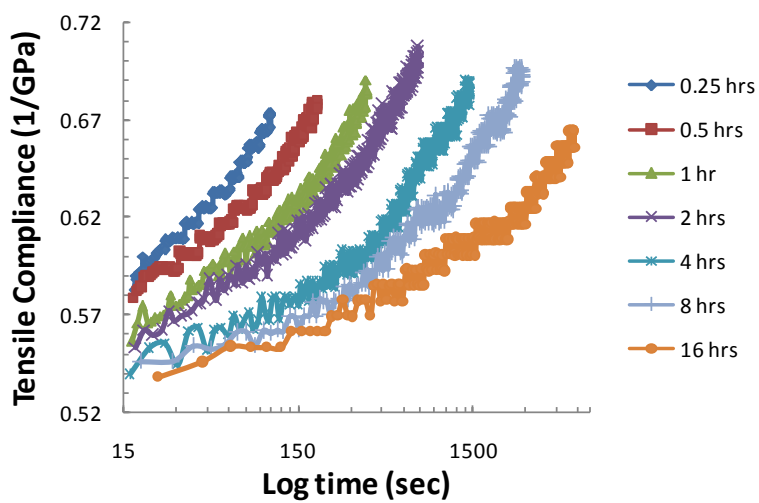

Figure 4. Compliance data for isothermal physical aging at $120^{\circ} \mathrm{C}$

\section{Analysis Of Results}

The curves shown in Fig. 4 have a lot of experimental noise and need to be smoothened before analysis. Kohlraush's functions are used to fit the experimental data and obtain smooth functions as shown in Fig. 5. The PHYAGE program was then run to calculate shift factors by taking the last curve (16 hrs) as the total reference curve. The data obtained from the PHYAGE program was shown in TABLE 1 where $D_{0}, \tau$ and $\beta$ are Kohlrausch parameters. The data shows how each creep compliance curve can be fit with a Kohlrausch function and the shift factors $\left(\mathrm{a}_{\mathrm{te}}\right)$ show the right trend. The shift factor is the factor that needs to be multiplied to the time of the creep test so that the curve can be shifted onto the total reference curve. In this case the total reference curve was set as the last set of data ( $16 \mathrm{hrs).} \mathrm{The} \mathrm{shift} \mathrm{rate} \mathrm{decreases} \mathrm{with} \mathrm{aging} \mathrm{time} \mathrm{and} \mathrm{the} \mathrm{shift} \mathrm{rate} \mathrm{for} \mathrm{the} 16$ $\mathrm{hr}$ curve is 1 as expected. This is because; the $16 \mathrm{hr}$ curve is the total reference curve and need not be shifted. The shift rate calculated from PHYAGE is 1.0238 for $120^{\circ} \mathrm{C}$.

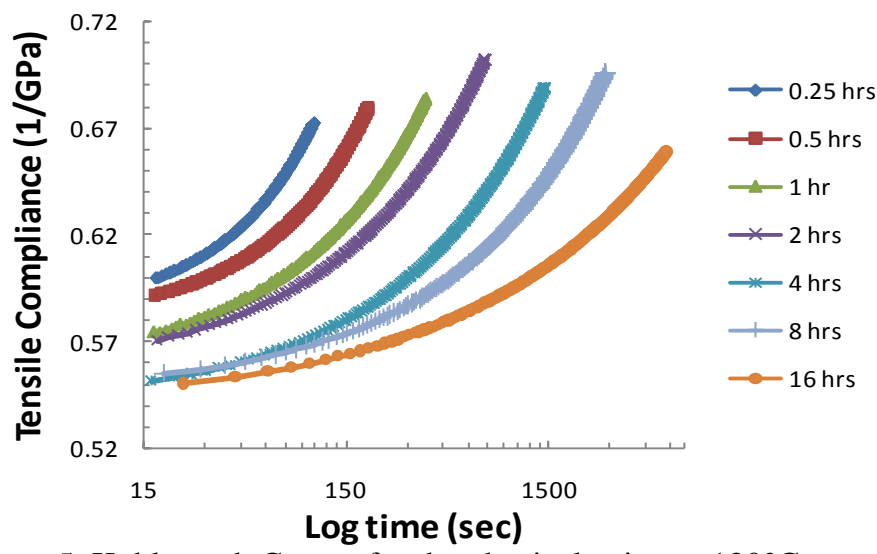

Figure 5. Kohlrausch Curves for the physical aging at $120^{\circ} \mathrm{C}$ 


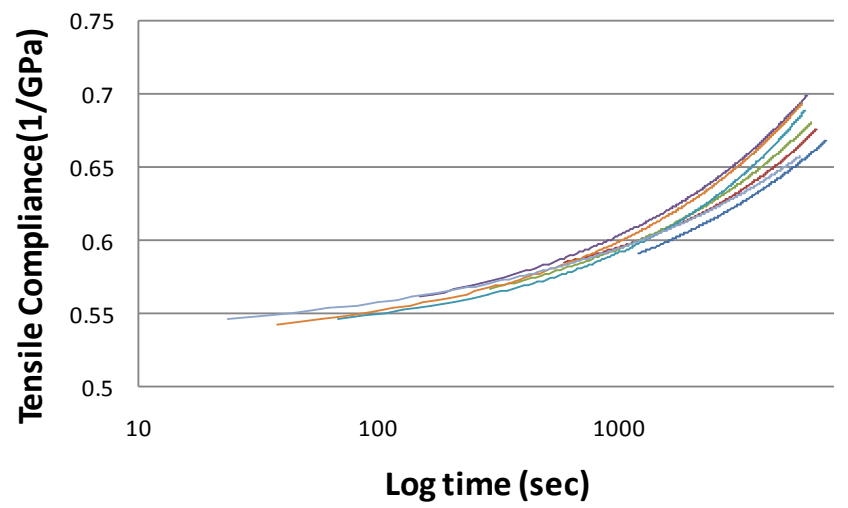

Fig. 6. Kohlrausch curve superposed on the total reference curve at $120^{\circ} \mathrm{C}$

Table 1. PHYAGE output for creep tests at $120^{\circ} \mathrm{C}$

\begin{tabular}{|c|c|c|c|c|}
\hline $\begin{array}{c}\text { Aging time } \\
(\mathrm{sec})\end{array}$ & $D_{O}(\mathrm{GPa})$ & $\tau(\mathrm{sec})$ & $\beta$ & $\begin{array}{c}\text { Shift factors } \\
\left(\mathrm{a}_{\mathrm{te}}\right)\end{array}$ \\
\hline $2.50 \mathrm{E}-01$ & $5.20 \mathrm{E}-01$ & $4.27 \mathrm{E}+03$ & $3.72 \mathrm{E}-01$ & $5.79 \mathrm{E}+01$ \\
\hline $5.00 \mathrm{E}-01$ & $5.54 \mathrm{E}-01$ & $4.04 \mathrm{E}+03$ & $5.30 \mathrm{E}-01$ & $3.64 \mathrm{E}+01$ \\
\hline $1.00 \mathrm{E}+00$ & $5.30 \mathrm{E}-01$ & $9.95 \mathrm{E}+03$ & $4.22 \mathrm{E}-01$ & $1.90 \mathrm{E}+01$ \\
\hline $2.00 \mathrm{E}+00$ & $5.30 \mathrm{E}-01$ & $1.57 \mathrm{E}+04$ & $4.17 \mathrm{E}-01$ & $1.24 \mathrm{E}+01$ \\
\hline $4.00 \mathrm{E}+00$ & $5.32 \mathrm{E}-01$ & $2.22 \mathrm{E}+04$ & $4.96 \mathrm{E}-01$ & $4.63 \mathrm{E}+00$ \\
\hline $8.00 \mathrm{E}+00$ & $5.25 \mathrm{E}-01$ & $5.91 \mathrm{E}+04$ & $4.23 \mathrm{E}-01$ & $2.72 \mathrm{E}+00$ \\
\hline $1.60 \mathrm{E}+01$ & $5.32 \mathrm{E}-01$ & $3.87 \mathrm{E}+05$ & $3.68 \mathrm{E}-01$ & $1.00 \mathrm{E}+00$ \\
\hline
\end{tabular}

The momentary curves multiplied by their respective shift factors should superpose the curve onto the total reference curve as shown in Fig. 6. The plot shows that the superimposition is not accurate. This is because the data at $16 \mathrm{hrs}$ has veered off from the other data sets and this data set was taken as the total reference curve. The removal of this data set or the selection of another data set as total reference curve would have yielded a better outcome. However, that is not attempted in this paper. The shifted factors thus calculated lie on a straight line when plotted on a log-aging time versus log shift factor graph as shown in Fig. 7 for $120^{\circ} \mathrm{C}$. Since the shift rate $(\mu)$ is obtained from PHYAGE, it can be used to draw the shift rate function that passes through the shift factors with a slope of $-\mu$. Similarly, the analysis is performed for creep results at $125^{\circ} \mathrm{C}$ and $130^{\circ} \mathrm{C}$. The shift rates at various temperatures as calculated by PHYAGE are shown in TABLE 2 . It can be observed that the shift rate value reduces with increasing temperature. The same can be corroborated from Fig. 8 where the slope of the shift rate function decreases with increasing temperature. This result is in line with the definition of Physical aging that states below $\mathrm{T}_{\mathrm{g}}$, the material gradually settles down in equilibrium. As the temperature goes further away from $\mathrm{T}_{\mathrm{g}}$, the rate of material transition towards stability increases.

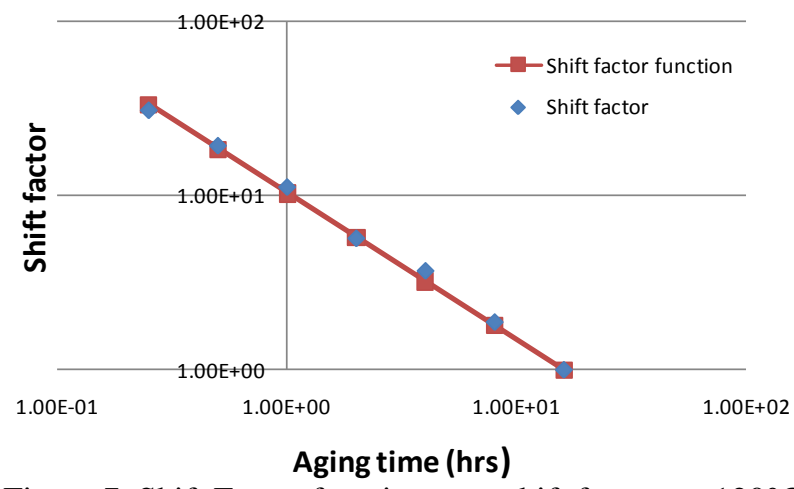

Figure 7. Shift Factor function over shift factors at $120^{\circ} \mathrm{C}$

Table 2. Shift rates at different temperatures

\begin{tabular}{|c|c|}
\hline $\begin{array}{c}\text { Test temperature } \\
\left({ }^{\circ} \mathrm{C}\right)\end{array}$ & Shift rate \\
\hline 120 & 1.0238 \\
\hline 125 & 0.844 \\
\hline 130 & 0.727 \\
\hline
\end{tabular}




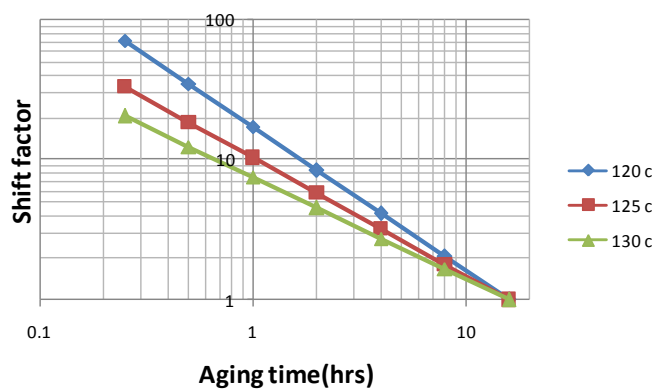

Figure 8. Comparison of shift rate functions obtained at various temperatures.

\section{Conclusion}

Polycarbonate material is tested by an established procedure to study the Physical aging behavior. A number of physical properties of the material are established.

- Firstly, the glass transition temperature $\left(\mathrm{T}_{\mathrm{g}}\right)$ was determined to be in range of $140-148^{\circ} \mathrm{C}$ by performing a dynamic temperature sweep test on a film specimen.

- Compliance data was obtained from the creep tests performed at 7 different aging times $-0.25 \mathrm{hrs}, 0.5$ hrs, $1 \mathrm{hr}, 2 \mathrm{hrs}, 4 \mathrm{hrs}, 8 \mathrm{hrs}$ and $16 \mathrm{hrs}$ and at various temperatures $-120^{\circ} \mathrm{C}, 125^{\circ} \mathrm{C}$ and $130^{\circ} \mathrm{C}$.

- Kohlrausch functions were used to characterize compliance data from different creep tests.

- PHYAGE program was successfully used to calculate the shift rate function, which characterizes the physical aging behavior at each temperature.

- The shift rate decreases with increasing temperature suggesting that at temperatures close to $T_{g}$, shift rate becomes negligible.

- The results follow an established pattern as described by Struik [1] and shows that as the aging temperature moves further away from $\mathrm{T}_{\mathrm{g}}$, the shift rate is faster for the material.

\section{Acknowledgements}

We would like to thank Dr. Roger Bradshaw from University of Louisville for his technical expertise and for providing the facilities required for this work.

\section{References}

[1] L.C.E. Struik, Physical Aging in Plastics and Other Glassy Materials, Polymer Engineering and science, 17(3), 1977, 165-173.

[2] L.C.E. Struik, Dependence of Relaxation-Times of Glassy-Polymers on Their Specific volume, Polymer, 29(8), 1988, $1347-1353$.

[3] John D. Ferry, Viscoelastic Properties of Polymers (3rd Edition, ISBN: 978-0-471-04894, 1980).

[4] J. L. Sullivan, Creep and Physical Aging of Composites, Composite Science and Technology, 39(3), 1990, $207-232$.

[5] E.N. Lawrence and F.L. Robert, Mechanical Properties of Polymers and Composites (Second edition, Marcel Dekker, New York, 1994).

[6] J. Z. Wang, H. Parvatareddy, T. Chang, N. Iyengar, D.A. Dillard, and K.L. Reifsnider, Physical Aging Behavior of HighPerformance Composites, Composites Science and Technology, 54(4), 1995, 405-415.

[7] L.C. Brinson and Tom.S. Gates, Effect of physical aging on long term creep of polymers matrix composites, International Journal of Solids Structures, 32(6-7), 1995, 827-846.

[8] R. D. Bradshaw, L.C. Brinson, Physical aging in polymers and polymer composites: An analysis and method for time-aging time superposition, Polymer Engineering and Science, 31(1), 1997, 31-44.

[9] P.A. O'Connell, G. B. McKenna: Large deformation response of polycarbonate, time-temperature, time-aging time, and time-strain superposition, Polymer Engineering \& Science, 37(9), 1997, 1485-1495.

[10] D.S. Matsumoto, Time-temperature superposition and Physical aging in amorphous polymers, Polymer Engineering \& Science, 28(20), 1998, 1313-1317.

[11] J.M. Hutchinson, S. Smith, B. Horne, and G. M. Gourlay, Physical Aging of Polycarbonate, Enthalpy Relaxation, Creep Response, and Yielding Behavior, Macromolecules, 32(15), 1999, 5046-5061.

[12] K.K. Biswas, M.Ikueda, S. Somiya, Study on creep behavior of glass fiber reinforced polycarbonate, Advanced Composite Materials, 10(2), 2001, 265-273.

[13] D. Cangialosi , H. Schut, A. Van Veen, and S.J. Picken, Positron Annihilation Lifetime Spectroscopy for Measuring Free Volume during Physical Aging of Polycarbonate, Macromolecules, 36(1), 2003, 142-147.

[14] A. Victor, C.M. Soloukhin, Jose Brokken-Zijp, Otto L. J. van Asselen, and Gijsbertus de, Physical Aging of Polycarbonate, Elastic Modulus, Hardness, Creep, Endothermic Peak, Molecular Weight Distribution, and Infrared Data, Macromolecules, 36(20), 2003, 7585-7597.

[15] E. Barbero, K. Ford, Determination of aging shift factor rates for field-processed polymers, Journal of Advanced Materials, 38(2), 2006, 7-13.

[16] Takenobu Sakai, Takayuki Tao, Satoshi Somiya, Viscoelasticity of Shape Memory Polymer, Polyurethane series DIARY, Journal of Solid Mechanics and Materials Engineering, 1(4), 2007, 480-489.

[17] Takenobu Sakai, Satoshi Somiya, Analysis of Creep Behavior in Thermoplastic based on Visco-elastic Theory, Mechanics of TimeDependent Materials, 15(3), 2009, 293-308.

[18] Takenobu Sakai, Satoshi Somiya, Effect of Thermal History on the Creep Behavior of Polycarbonate, Journal of Solid Mechanics and Materials Engineering, 3(11), 2009, 1193-1201.

[19] R. R. Resapu, Isothermal and Non-isothermal aging of polymers, Master's Thesis, University of Louisville, Louisville, KY, 2005. 